\title{
Semantic Techniques of Image Retrieval - The Example of a Structural Analysis of Coronary Arteries
}

\author{
Mirosław Trzupek and Marek R. Ogiela \\ AGH University of Science and Technology, \\ Department of Automatics and Biomedical Engineering, \\ al. A. Mickiewicza 30, 30-059 Krakow \\ \{mtrzupek, mogiela\} @agh.edu.pl
}

\begin{abstract}
Specialised medical databases currently play a major role in archiving and searching for comparable data acquired by different modalities of medical imaging. In the context of storing and searching for data in medical databases, it is of immense importance to find a method of extracting and representing the contents found in the image that would ensure the rapid access and satisfactory results of searches for image information records. What is important, this representation should also be independent of the form of the image. The wide spread of multimedia medical databases that can store not just single images but also video sequences has shown that the problem of effectively searching for images containing specific disease cases that are significant for medical diagnostics is still fraught with great difficulties. This article presents a semantic retrieval methods in medical imaging databases using graph formalisms of syntactic image recognition which contribute to solving these problems. The proposed methods although they are mainly predestined for medical applications can also provide a base for other solutions, particularly for the acquisition and sophisticated semantic analysis of complex image patterns for security and defence reasons.
\end{abstract}

Keywords: Semantic image retrieval, content-based image retrieval (CBIR), image understanding systems.

\section{Introduction}

In recent years, digital images have become a constant part of our life, while in the field of medical imaging they significantly contribute to saving this life. It is hard to imagine making a medical diagnosis and treating many disorders without such basic medical imaging apparatuses as X-ray, CT, USG or MRI equipment. Image data obtained by various medical imaging modalities makes it possible to non-invasively look into the patient's body, which means that the popularity of such diagnostic methods is constantly increasing. On the other hand, the increasing availability of medical imaging apparatuses and, as a direct result, the rapidly growing set of images generated by these apparatuses lead to a major glut of image data used in medical diagnostics. In order to fully utilise its potential, this data must be stored in the right 
way, particularly so that it is easily accessible. In this context, it is worth noting that proposed methodology may also be used in the area that combines security and defence aspects in designing advanced systems for the retrieving, acquisition, storing and sophisticated semantic analysis of complex image patterns and group behaviours. In the case of medical diagnostics, this field is currently dominated by systems for archiving medical image data, such as PACS (Picture Archiving and Communication Systems). In particular, these systems are now responsible for the correct and secure transmission, storage and retrieval of image data. The way in which single image is searched in a huge set of image data collected in these databases is the major weakness of these systems. Most frequently, this data is searched for by filling out the appropriate fields with search criteria in the form of alphanumeric data (text and numbers), such as: the patient's personal data, the examination date, the examination description or the selection of the appropriate image modality. Figure 1 shows a typical screen shot from a PACS system.

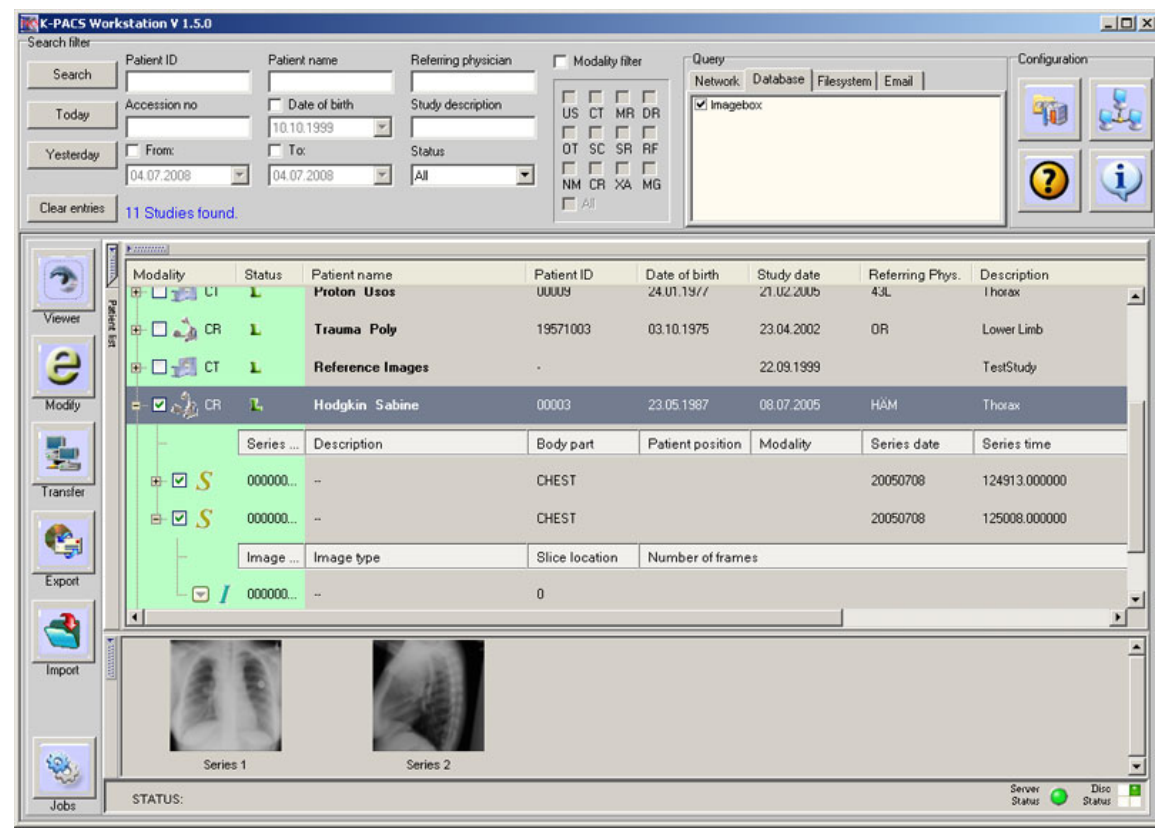

Fig. 1. The main window of the K-PACS system supporting viewing a medical database and searching in it [source: K-PACS V1.6.0 DICOM Viewing Software]

The search criteria are entered as attributes in the alphanumeric format mainly because the images stored in such specialised databases also contain alphanumeric information (describing these images) saved, inter alia, in the headers of the archived files (e.g. DICOM files). Certain inaccuracies or ambiguities in the descriptions are more probable where human factor comes into play during inputting the search information. Those inaccuracies may lead to incorrectly assigning the description to a given image what, in extreme cases, leads to increasing the risk of mistakes. 
In addition, describing images textually is labour- and time-intensive, and in the case of medical images requires great experience in interpreting their contents, thus posing a risk of important information being omitted in the description. On the other hand, the clear upside of this method of medical data storage is that image information records of interest can be relatively quickly and effectively found in huge resources of specialised medical databases.

\section{Development of Systems for Context-Indexing Images}

Systems for indexing images with their contents date back to the 1970s, but very intensive work on developing such systems was done in 1990s and resulted from the rapidly growing quantity of digital image data which made it necessary to look for effective methods of storing, indexing and accessing it [1,2]. Another, no less important factor was the rapid development of computer technology, which, in medicine, directly contributed to the design of new, increasingly advanced medical diagnostic apparatuses. This trend could be observed both in the area of professional equipment (e.g. used in medical imaging) and in everyday life (all kinds of devices for capturing images). In addition, the constantly rising computing power of computers made it possible to undertake problems of digital image analysis which could not be solved before due to their high computational complexity (e.g. morphological transformations). The rapid development of computer methods of digital image analysis and processing which started then has now led not only to the accumulation of a huge quantity of image data, but also to elaborating many algorithms for the computer analysis and processing of images. This means that the great volume of data kept in specialised medical databases requires the creation of increasingly effective algorithms for indexing and finding specific cases.

The traditional approach to managing resources of image data is based on indexing images with their alphanumeric descriptions, but if the sets of this type of data are huge, problems arise because the search results are often very far from optimal. This is mainly due to the vast amount of data that can be contained in an image (this is obvious particularly in the case of medical images), as a result of which even the best verbal description cannot fully present the contents of a given image ("a picture is worth a thousand words"). Neither are descriptions using key words of much help, regardless of their incontrovertible advantage stemming from their brevity. This is mainly due to the fact that the elements of interest (areas of interest) of the image greatly depend on the context, so key words that can be used to describe a given image may be different and not necessarily as expected. This demonstrates the importance of researches in the field of image managing and predefined criteria based searching algorithms. What helps in this regard are CBIR (content-based image retrieval) systems based on the idea of finding images according to their contents. The technique most frequently used in these systems is the query by image content (QBIC), in which the user inputs (or draws) an example image, and the system finds images kept in the database that are similar to this set pattern. CBIR systems such as 
the QBIC [3] allow images retrieving according to their visual features such as e.g. their colour, texture, and shape features, etc. The interface of a typical CBIR system is shown in Figure 2.

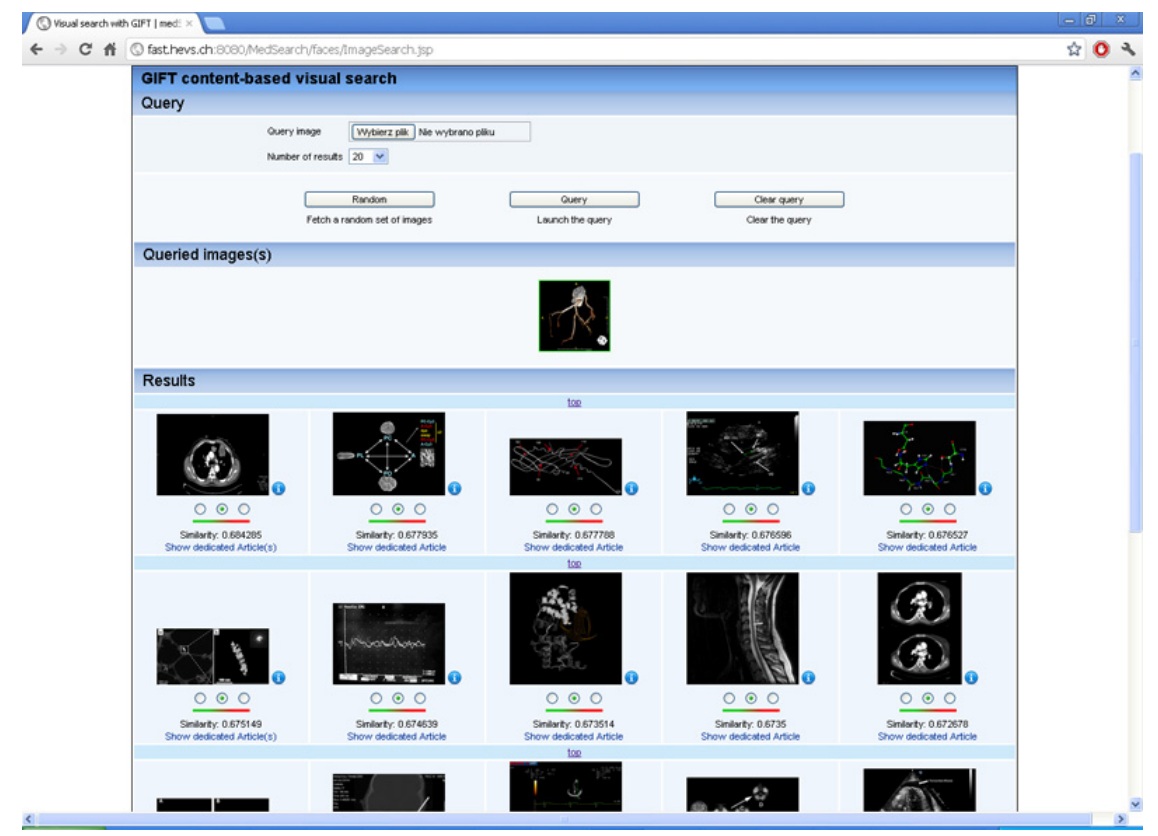

Fig. 2. A screen shot of the MedSearch interface [source: MedGIFT Content-based medical image retrieval]

Another, more refined method of searching within image data sets consists in an attempt to explore the contents of the image using its semantic characteristics (e.g. the type of object or event shown in the image). This is of major importance for medical images, as in their specific case, the computer has to try to explore the meaning of the lesions observed in the image and not just to analyse their form. This 2 level distinction (primitive image features and semantic features) is introduced in article [4] in connection with looking for effective methods of automatically retrieving, archiving and semantically categorising image data in multi-media databases. The author of article [5] go a step further and distinguish 3 levels (primitive features, logical features, abstract attributes), which, in addition, can then be split into more sub-levels.

This article presents methods of semantic image retrieval in medical databases using semantic features of images. There are still a number of unsolved problems associated with the subject defined above, so the authors are presenting a proposal that applies to a certain class of images, specifically images of the coronary vascularisation obtained from diagnostic examinations with the use of computed tomography (CT). 


\section{Technologies of Semantic Image Retrieval - Example of CT Images of Coronary Vascularisation}

This paper presents the possibility to use graph formalisms of mathematical linguistic to organise, index and support semantically finding and selecting image information. The set of image data consists of images originating from computed tomography (CT) diagnostics. The semantic approach to indexing and searching databases is more effective than traditional retrieval methods, and the search results produced are more correlated with the set pattern in terms of the contents. This is, of course, due to the descriptive capacity of the semantic methods used, which allow images to be grouped semantically, i.e. not according to their form, but to their contents. The methods presented apply to medical images. It has already been suggested that for this image class, it is very important to extract semantic features and then use them to manage sets of image data because images containing similar diagnostic information about the disease process frequently have completely different forms. In such images, the specific shapes of the lesions observed may take many forms due to individual differences between the diagnosed patients.

The presented methodology of automatically creating semantic descriptions of images stored in multi-media medical databases is based on methods of semantically interpreting coronary arteries, successfully used to describe and identify lesions in coronary vascularisation images as part of previous studies by the authors [6-9]. What is important in creating sequences describing images from a database is a method of effectively transforming the image information contained in these images (which is easily perceived by a human) to a machine format which supports the intelligent, semantic selection of a specific case (easily assimilated by a computer). One possible method consists in the proposed grammar formalisms for the structural analysis of images, in which the analysed image is treated as a hierarchical structure composed of so-called picture primitives. In their previous publications, the authors proposed using graph grammars to describe and model the spatial relations of coronary vascularisation reconstructions [6-9]. Grammars of this type generate a formal language in the form of graphs which can model the images considered here, and then the graphs obtained can be represented in the form of their characteristic descriptions. So the mechanism presented makes it possible to transform image information contained in images into a machine format, namely a characteristic description of graphs modelling the coronary vascularisation. What is more, this description can be additionally complemented by sequences generated by sequential grammars [9] that are used to represent the width graphs of individual coronary vessels (represented by the edges of the graph modelling the coronary vascularisation). Of course, the use of grammar formalisms also offers a number of other possibilities available when indexing with the use of the grammars applied. Apart from characteristic descriptions representing the graph modelling the given structure enhanced with sequences generated by sequential grammars, it is also possible to use mechanisms provided by the introduced grammar, e.g. in the form of a sequence of numbers of derivation rules of this grammar, defined by the set of their productions. The general diagram of retrieving images from a semantically indexed specialised medical database with the use of the above methodology is presented in Figure 3. 


\begin{tabular}{|c|c|c|c|c|c|}
\hline \multicolumn{6}{|c|}{ SPECIALISED MEDICAL DATABASE } \\
\hline P ID & $\begin{array}{l}\text { Patient } \\
\text { data }\end{array}$ & $\cdots$ & $\begin{array}{l}\text { Description of the } \\
\text { individual case }\end{array}$ & $\begin{array}{l}\text { Semantic description } \\
\text { of the image contents }\end{array}$ & Image \\
\hline$\# 12 \mathrm{AB}$ & Smith... & 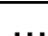 & IIIness description & $\alpha \beta \gamma \delta \varepsilon \zeta \eta \theta 1 \kappa \lambda \mu v \xi о \pi \rho \varsigma . .$. & Q \\
\hline \multicolumn{6}{|c|}{ 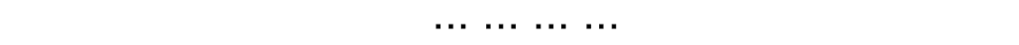 } \\
\hline & & & & & \\
\hline
\end{tabular}
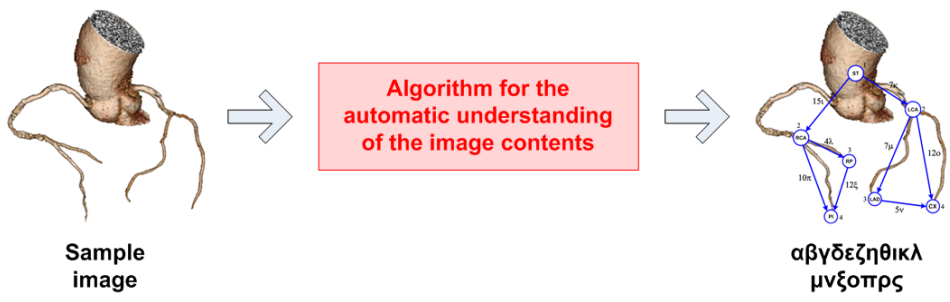

Fig. 3. Using the above methodology to search for information in a semantically-indexed specialised medical database by reference to a set pattern

In this case, semantic searching boils down to comparing the sequence of semantic descriptions representing the contents of individual images stored in the database to the sequence representing the semantic description of the set pattern (the semantic description of the input image which constitutes the set pattern must also be semantically described in accordance with the presented methodology). As the mechanism thus defined should generate results which accurately correspond to the set pattern, there is a risk that these results will include only a few images. To avoid this, a certain threshold of similarity between the semantic description sequence representing the data in the database and the sequence representing the set pattern should be defined. Although this will increase the risk of obtaining results that are not fully satisfactory, it will also raise the number of results similar to the set pattern, which number can be controlled by adjusting the similarity threshold as necessary. In the case of medical images, additional similar results may help the physician in his/her work. The formal definition of the similarity threshold of sequences of indexing keys will form the subject of further research.

To conclude, it is worth noting that in the case of medical databases, a structural description of image contents with the use of the presented linguistic formalisms is more unambiguous than the traditional description methods using e.g. the colour or texture. This is significant because it has direct impact on the results of searches for images similar to the set pattern in multi-media databases, thus producing more 
results which strongly correlate with the set pattern in terms of the contents of these images.

\section{Summary and Further Research Directions}

Image indexing systems currently in use have several major shortcomings, and therefore cannot be fully utilised. First of all, the majority of them is based on an alphanumeric description written by a human, which can be imperfect. It frequently omits information that is significant from an objective point of view, and often contains errors. Also the headers of DICOM files can contain a relatively high proportion of errors [11], and this can hinder the correct retrieval of all the images looked for. What is more, systems whose operation is based on visual characteristics can usually process low-level attributes (the colour, texture, shape etc.) without being able to identify high-level features (relationships between objects, object types etc.), or they are able to do this only for a very narrow class of images.

Here it should also be stressed that medical images constitute a special class of images in which images completely different graphically may have the same contents, so particularly for these images there is a need of tools that can correctly extract the image contents masked by varied forms. A doctor following diagnostic guidelines frequently wants to review images showing a similar disorder so that the decision he/she takes is based on many premises and is therefore optimal. In this situation, the attempt to find an image that is similar (in terms of its contents, not form) in large alphanumerically indexed databases leads to retrieving a large number of images whose contents may not be correlated with the set pattern. Hence it is obvious that providing the doctor with the appropriate tools allowing databases to be semantically indexed using an example image pattern can contribute to improving the precision of that physician's diagnostic decisions. In this context, the methods of semantically indexing and retrieving images from medical databases, presented in this article using the example of CT images of coronary vascularisation, significantly contribute to solving at least some of the problems associated with the effective storage, indexing and access to this data. It is also obvious that the proposed mathematical linguistic formalisms offer great potential. Of course, the solutions proposed apply only to a selected class of diagnostic images showing coronary vascularisation, but it is possible to adapt the above solutions to another class of images, especially for complex image patterns (e.g. research that combines security and defence aspects) [12].

It is also worth noting that the solutions described in the literature are frequently dedicated only to a narrow class of images or are limited only to a specific database [13]. An ideal system would be able to combine these databases and search through a much greater number of available sources of medical images. What is more, sometimes a system, even though dedicated to a broader group of images, does better with patterns from one class and worse with those from another class for which the implemented mechanisms are less suitable. These and many other problems discovered during research on such systems will also form the subject of further intense work by the authors. 
Acknowledgments. This work has been supported by the Ministry of Science and Higher Education, Republic of Poland, under project number N N516 478940.

\section{References}

1. Eakins, J.P.: Towards intelligent image retrieval. Pattern Recognition 35, 3-14 (2002)

2. Müller, H., Michoux, N., Bandon, D., Geissbuhler, A.: A review of content-based image retrieval systems in medical applications-clinical benefits and future directions. I. J. Medical Informatics 73(1), 1-23 (2004)

3. Niblack, W.R., et al.: The QBIC project: querying images by color, texture and shape. IBM Research Report RJ-9203 (1993)

4. Gudivada, V.N., Raghavan, V.V.: Content-based image retrieval systems. IEEE Comput. 28(9), 18-22 (1995)

5. Eakins, J.P.: Techniques for image retrieval. Library and Information Briefings 85, British Library and South Bank University, London (1998)

6. Ogiela, M.R., Tadeusiewicz, R., Trzupek, M.: Picture grammars in classification and semantic interpretation of $3 \mathrm{D}$ coronary vessels visualizations. Opto-Electronics Review 17(3), 200-210 (2009)

7. Trzupek, M.: Semantic Interpretation of Heart Vessel Structures Based on Graph Grammars. In: Bolc, L., Tadeusiewicz, R., Chmielewski, L.J., Wojciechowski, K. (eds.) ICCVG 2010, Part I. LNCS, vol. 6374, pp. 81-88. Springer, Heidelberg (2010)

8. Trzupek, M.: Linguistic approach in intelligent image content analysis of heart vessel structures. In: Barolli, L., et al. (eds.) The Fourth International Conference on Complex, Intelligent and Software Intensive Systems, CISIS 2010, February 15-18, pp. 856-859. IEEE Computer Society, Krakow (2010)

9. Trzupek, M., Ogiela, M.R., Tadeusiewicz, R.: Intelligent image content semantic description for cardiac 3D visualizations. Engineering Applications of Artificial Intelligence 24, 1410-1418 (2011)

10. Poisel, R., Tjoa, S., Tavolato, P.: Advanced File Carving Approaches for Multimedia Files. Journal of Wireless Mobile Networks, Ubiquitous Computing, and Dependable Applications 2(4), 42-58 (2011)

11. Güld, M.O., Kohnen, M., Keysers, D., Schubert, H., Wein, B.B., Bredno, J., Lehmann, T.M.: Quality of DICOM header information for image categorization. In: Proceedings of the International Symposium on Medical Imaging, San Diego, CA, USA, vol. 4685, pp. 280-287 (2002)

12. Wolter, P.: Representing humans in system security models: An actor-network approach. Journal of Wireless Mobile Networks, Ubiquitous Computing, and Dependable Applications 2(1), 75-92 (2011)

13. Ghosh, P., Antani, S., Long, L.R., Thoma, G.R.: Review of medical image retrieval systems and future directions. In: CBMS, pp. 1-6 (2011) 